\title{
8
}
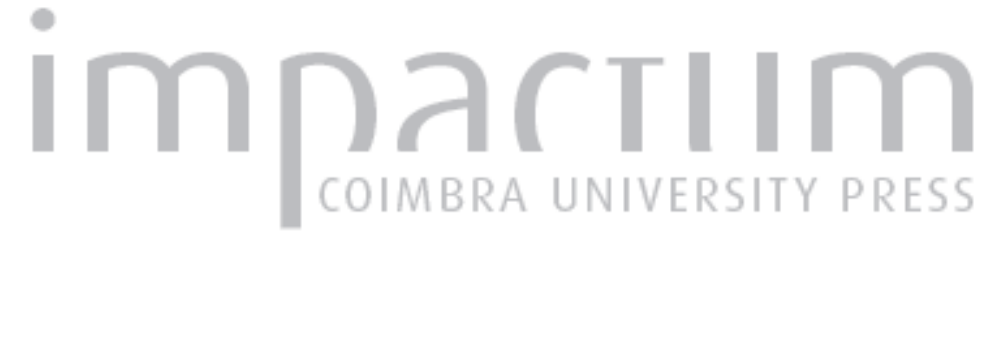

\section{Vulnerabilidade à contaminação de águas subterrâneas: comparação de modelos baseados no método DRASTíc}
Autor(es):
Carvalho, Gonçalo

Publicado por:

Faculdade de Letras da Universidade de Coimbra, Departamento de Geografia

URL

persistente:

URI:http://hdl.handle.net/10316.2/30257

DOI:

DOI:http://dx.doi.org/10.14195/0871-1623_32_24

Accessed : $\quad$ 26-Apr-2023 13:53:40

A navegação consulta e descarregamento dos títulos inseridos nas Bibliotecas Digitais UC Digitalis, UC Pombalina e UC Impactum, pressupõem a aceitação plena e sem reservas dos Termos e Condições de Uso destas Bibliotecas Digitais, disponíveis em https://digitalis.uc.pt/pt-pt/termos.

Conforme exposto nos referidos Termos e Condições de Uso, o descarregamento de títulos de acesso restrito requer uma licença válida de autorização devendo o utilizador aceder ao(s) documento(s) a partir de um endereço de IP da instituição detentora da supramencionada licença.

Ao utilizador é apenas permitido o descarregamento para uso pessoal, pelo que o emprego do(s) título(s) descarregado(s) para outro fim, designadamente comercial, carece de autorização do respetivo autor ou editor da obra.

Na medida em que todas as obras da UC Digitalis se encontram protegidas pelo Código do Direito de Autor e Direitos Conexos e demais legislação aplicável, toda a cópia, parcial ou total, deste documento, nos casos em que é legalmente admitida, deverá conter ou fazer-se acompanhar por este aviso. 


\section{Vulnerabilidade à contaminação de águas subterrâneas: comparação de modelos baseados no método DRASTIC}

\section{Gonçalo Carvalho}

Doutorando em Geografia Física na Faculdade de Letras da Universidade de Coimbra. gjpcarvalho@gmail.com

\section{Resumo:}

A bacia hidrográfica do rio Sordo foi estudada relativamente à sua vulnerabilidade face à eventual contaminação das suas águas subterrâneas, sendo para tal utilizado o modelo DRASTIC apresentado por ALLER et al. (1985), determinado por sete parâmetros, com importância diversa. Devido à sua característica subjetiva aplicou-se uma modelação fuzzy do "Padrão de Reconhecimento Multi-Objectivos" desenvolvido por ZHou et al. (1999), que aplicada ao índice DRASTIC tenta ilustrar que a rigidez dos limites aplicados aos diferentes parâmetros de avaliação da vulnerabilidade. Foi ainda aplicado à mesma bacia o método da concentração em nitratos na avaliação da vulnerabilidade à poluição dos sistemas aquíferos, através da adopção da metodologia desenvolvida por Antonakos e Lambrakis (2007).

Palavras-chave: Vulnerabilidade. DRASTIC. Fuzzy. Concentração em Nitratos. Rio Sordo.

\section{Résumé:}

La vulnérabilité à la contamination des eaux souterraines: la comparaison des modèles basés sur la méthode DRASTIC

Le bassin hydrographique Sordo a été étudiée par rapport à sa vulnérabilité à la contamination possible de leurs eaux souterraines et il est donc utilisé le modèle DRASTIC présenté par ALLER et al. (1985) déterminée pour sept paramètres qui ont une importance différente. Grâce à sa caractéristique subjective appliqué une modélisation fuzzy (floue), «Pattern Recognition Multi-Objectifs» développé par Znou et al. (1999), qui s'applique à l'indice DRASTIC tente d'illustrerque la rigidité des limites appliquées à des paramètres différents pour évaluer la vulnérabilité. Il a également été appliquée à un même bassin, la méthode de concentration de nitrates dans l'évaluation de la vulnérabilité à la pollution des aquifères par l'adoption de la méthodologie développée par Antonakos e Lambrakis (2007).

Mots-clés: Vulnérabilité. DRASTIC. Fuzzy (floue). Concentration de nitrate. Rivière Sordo.

\section{Abstract:}

Vulnerability of underground aquifers to contamination: DRASTIC method based models comparison.

The hydrographic basin of Sordo river was studied for its vulnerability resulting from the eventual contamination of their underground waters, using the DRASTIC model presented by ALLER et al. (1985), determined by seven parameters, with different weights. Due to its subjective characteristics, a fuzzy modulation was applied by the "multi-objective fuzzy pattern recognition model" developed by Zнои et al. (1999), which when applied to the DRASTIC model, tries to show its parameters stiffness for vulnerability evaluation. To the same basin was also applied the "Nitrate Concentration Model" to review the aquifer system vulnerability to pollution, and the chosen methodology was developed by ANTONAKOS e LAMBRAKIS (2007).

Keywords: Vulnerability. DRASTIC. Fuzzy. Nitrate Concentration. Sordo river. 


\section{Introdução}

O método DRASTIC é o mais vulgarmente utilizado para avaliar a sensibilidade aquífera (USGS, 1999), e foi aplicado por muitos autores, em diversos estudos e variadas áreas espalhadas por todo o Globo

A avaliação da vulnerabilidade dos recursos hídricos subterrâneos à contaminação pelo método DRASTIC (Aller et al., 1987) é determinada por sete parâmetros, com importância diversa, identificados por cada uma das letras do acrónimo DRASTIC a que correspondem a profundidade do topo do aquífero ( $D$, peso 5$)$, a recarga do aquífero $(R, 4)$, a tipologia do material do aquífero $(A, 3)$, o tipo de solo $(S, 2)$, a topografia $(T, 1)$, o impacto da área não saturada ou vadosa (I, 5), e a condutividade hidráulica do aquífero $(C, 3)$. A contribuição de cada parâmetro para a vulnerabilidade global é diferente sendo expressa através de pesos (w). Segundo Antonakos e Lambrakis (2007), a vulnerabilidade global (DRASTIC) é definida por uma equação linear depois de reduzidos os limites físicos a uma escala relativa com valores entre um e dez. Cada parâmetro é multiplicado por um coeficiente - peso - que foi determinado subjectivamente, baseado no julgamento profissional dos autores deste método. A equação linear que determina a metodologia é a seguinte:

$D R A S T I C=D \times w D+R \times w R+A \times w A+S \times W S+T \times w T+\mid \times w I+$ $C \times W C$

A principal fragilidade do modelo DRASTIC consiste pois na subjetividade inerente à conversão das escalas físicas na escala de 10 categorias e na atribuição dos pesos relativos a cada parâmetro. Outras debilidades têm sido apontadas estando relacionadas com o porquê da inclusão de certos parâmetros em detrimento de outros (Antonakos e Lambrakis, 2007).

Os modelos de avaliação ou decisão genericamente designados por fuzzy surgem normalmente como ferramentas de aperfeiçoamento de determinada metodologia. Aplicada ao índice DRASTIC, a modelação fuzzy tenta ilustrar que a rigidez dos limites e pesos aplicados aos diferentes parâmetros de avaliação da vulnerabilidade não será o fim da pesquisa, surgindo os métodos fuzzy como complementos a uma investigação mais profunda, que se adapta e transforma consoante as particularidades do meio que se estuda. Ligados à tradução do termo fuzzy aparecem os termos indistinto, confusão ou mistura.

Introduzidas por ZADEH (1965), a lógica fuzzy e a teoria fuzzy têm sido utilizadas em modelos de tomada de decisão ambíguos e incertos. 0 conceito básico da lógica fuzzy é simples: as afirmações não são só verdadeiras ou falsas; na teoria, ser parcialmente verdadeiro e falso em simultâneo também é possível, em proporções definidas pelas designadas funções membro (AfSHAR et al., 2007).

Vários autores aplicaram modelos fuzzy com fins diversos, adaptando a sua metodologia ao caso que estavam a estudar, do que resultou a criação de novos métodos baseados nas características indistintas das propriedades físicas dos locais em investigação. Por exemplo, ZHou et al. (1999) verificaram o funcionamento de um "Padrão fuzzy multi-objectivos", aplicado ao índice DRASTIC, na área nordeste da China.

O modelo DRASTIC estabelece a vulnerabilidade intrínseca do meio à contaminação, não existindo muitos trabalhos que aproximem o método a uma análise de risco, embora em alguns casos se procure validar os resultados do DRASTIC através do cálculo de correlações entre os valores do DRASTIC e indicadores de poluição (KALINSK et al., 1994; RUPERT, 1999 in USGS; MCLAY et al., 2001).

No presente estudo procede-se ainda à determinação do risco de poluição na bacia do rio Sordo, utilizando para tal uma adaptação do modelo proposto por ANTONAKOS e LAMBRAKIS, 2007, derivado do DRASTIC, designado "Modelo da Concentração em Nitratos". As modificações essenciais relativamente ao modelo original propostas por este autor consistem na adição do parâmetro "Uso do Solo" (parâmetro L) como variável indicadora da vulnerabilidade específica do meio; na revisão das escalas de cada um dos parâmetros DRASTIC, incluindo o parâmetro $\mathrm{L}$, tomando em consideração as concentrações em nitratos observadas ou estimadas para a região em estudo; e na revisão dos pesos atribuídos a cada parâmetro com base nas correlações estabelecidas entre os valores dos mesmos e as concentrações em nitratos observadas ou estimadas para a região em estudo. 0 método da concentração em nitratos foi desenvolvido com base nos seguintes pressupostos: a) as águas subterrâneas de uma determinada região apresentam um risco potencial de serem contaminadas que é determinado pela seguinte relação: RISCO POTENCIAL = VULNERABILIDADE INTRÍNSECA + VULNERABILIDADE ESPECÍFICA

Em que a vulnerabilidade intrínseca é determinada pelos 7 parâmetros do modelo DRASTIC na sua versão original e a vulnerabilidade específica é determinada pelo uso do solo. A vulnerabilidade específica está associada aos diversos usos do solo, de acordo com os critérios estabelecidos por Antonakos e LAMBRAKIs (2007). 0 peso atribuído por este autor à vulnerabilidade específica é 4 . 0 risco real é o que pode ser aferido num momento específico comparando o risco potencial com as concentrações em nitratos observadas nesse mesmo momento. No caso das concentrações em nitratos exce- 
derem os limites legais, os índices de risco desses locais correspondem a índices de contaminação efectiva.

\section{2. Área de estudo}

A bacia hidrográfica do Rio Sordo localiza-se no Norte de Portugal, mais precisamente no distrito de Vila Real. Integra-se na extensa bacia hidrográfica do rio Douro, ocupando uma superfície com cerca de 50 $\mathrm{km}^{2}$ e sendo atravessada pelo IP4 e pelas Estradas Nacionais N15 e N304. Os núcleos da população residente encontram-se dispersos por toda a área da bacia e, em termos altimétricos a bacia hidrográfica varia desde os 185 até os 1300 metros (Figura 1), possuindo declives por vezes superiores a $30 \%$ nas extremidades da bacia, sendo a área central pouco inclinada.
Sob o ponto de vista geológico (Figura 2), afloram na área rochas cristalinas em que dominam metassedimentos Paleozóicos intruídos por granitos Hercínicos, estes com expressão na área mais a jusante da bacia hidrográfica. Os depósitos recentes têm uma representação significativa na área de vale, constituindo uma mancha com cerca de $3,2 \mathrm{~km}^{2}$. Na área de contacto entre os granitos e os metassedimentos são frequentes intrusões filoneanas de aplitos cuja orientação é predominantemente NW-SE. Este dique aplítico serviu de barreira à drenagem do vale da Campeã, favorecendo a acumulação dos depósitos coluviais-aluviais provenientes dos cumes do Marão e do Alvão. São ainda frequentes filões de quartzo, de direcção variável, que surgem dispersos nas formações de rochas cristalinas. A fracturação é intensa salientando-se como dominantes o sistema com direcção NE-SW a NNESSW e o sistema conjugado NW-SE.

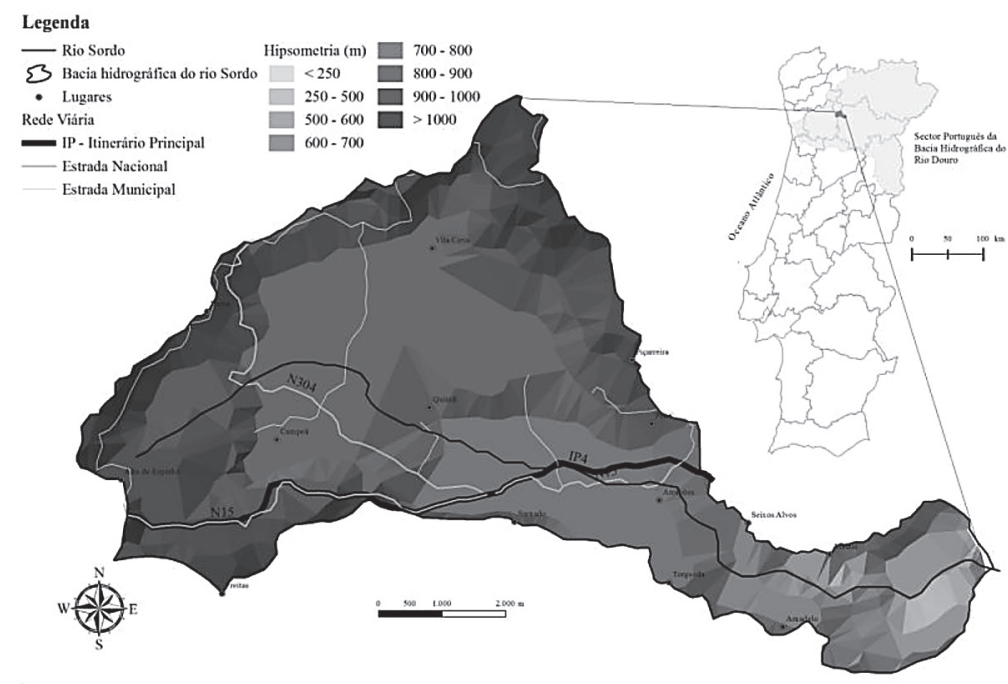

Figura 1

Enquadramento geográfico da bacia hidrográfica do rio Sordo.

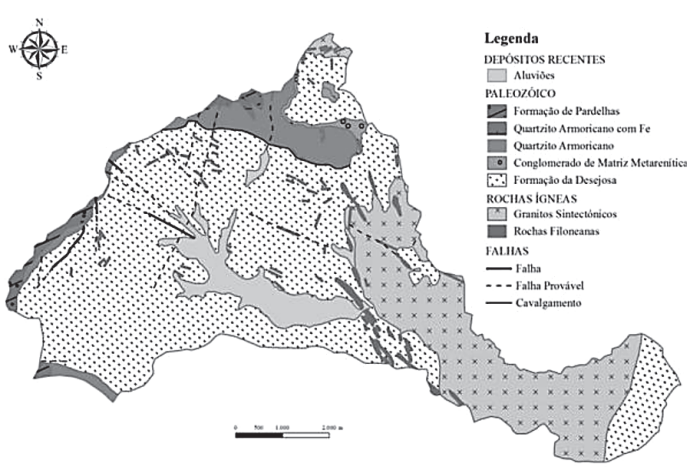

Figura 2

Enquadramento geológico da bacia hidrográfica do rio Sordo.

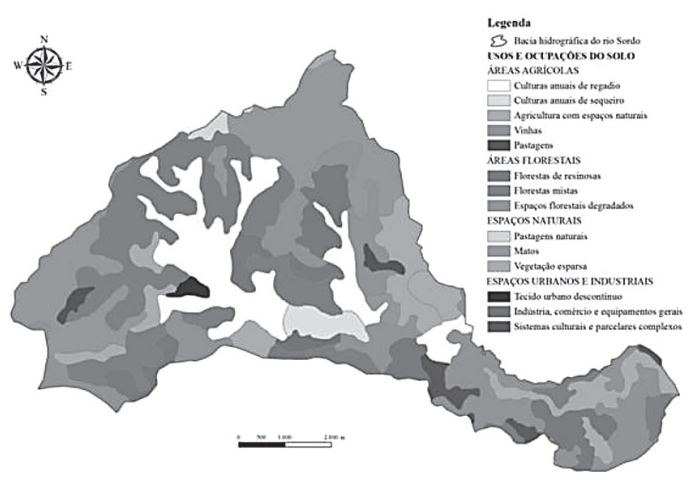

Figura 3

Mapa de uso do solo da bacia hidrográfica do rio Sordo. 
De acordo com Agroconsultores e Coba (1991), os tipos de solo que cobrem a maior parte da bacia hidrográfica do rio Sordo são os leptossolos $\left(42,3 \mathrm{~km}^{2}\right)$, surgindo com menor representação, na área do vale da Campeã, os fluvissolos $\left(5,9 \mathrm{~km}^{2}\right)$, e no sector mais a jusante da bacia hidrográfica os antrossolos $\left(3,0 \mathrm{~km}^{2}\right)$. As áreas urbanas ocupam cerca de $0,5 \mathrm{~km}^{2}$. 0 mapa de uso do solo da bacia hidrográfica do rio Sordo apresenta-se na Figura 3, podendo distinguir-se quatro tipos principais de ocupações: áreas agrícolas, caracterizadas por áreas destinadas a culturas de regadio ou sequeiro, por vinhas e pastagens, e por áreas onde a agricultura forma um mosaico integrado com espaços naturais; áreas florestais, constituídas essencialmente por manchas de resinosas ou mistas, por vezes sujeitas a desbastes e replantações; espaços naturais, que integram fundamentalmente os lameiros, os matagais e as áreas de vegetação esparsa ou rocha nua; espaços urbanos e industriais, dominados pelos povoados dispersos da região, por vezes numa estrutura complexa de aglomerados rodeados ou intervalados por áreas agro-florestais, e pelos equipamentos industriais da Campeã.

Os sistemas aquíferos dominantes são de tipo fissural e dependem, naturalmente, da fracturação e alteração dos afloramentos. Os depósitos de cobertura, pela elevada produtividade, poderão constituir bons sistemas aquíferos. As espessas camadas de alteração, que se associam em particular às rochas metassedimentares, originam sistemas hidrogeológicos com elevado potencial de armazenamento, constituindo aquíferos subsuperficiais significativos que servem também de elementos de recarga profunda (PACHECO et al, 2004).

\section{Metodologia}

\section{1. $O$ índice DRASTIC}

A primeira etapa resume-se à escolha do tipo de dados, os quais podem ser do tipo pontos, linhas e áreas, ou uma combinação destes. Esta escolha varia consoante o indicador hidrogeológico que se pretende caracterizar, o qual depende do parâmetro que se está a estudar. Depois de se estabelecer a tipologia dos dados considerados adequados a uma avaliação da vulnerabilidade DRASTIC, prossegue-se para a fase de aquisição dos mesmos e em sequência ao seu processamento para que possam ser objecto de posterior conversão em índices parcelares DRASTIC.

O passo subsequente à aquisição e organização dos dados em bruto consiste na quantificação e qualifi- cação dos indicadores hidrogeológicos em que assenta o modelo DRASTIC. Realizada esta tarefa, surge uma outra que consiste na reclassificação dos indicadores hidrogeológicos na forma de índices parcelares DRASTIC, ou seja, na preparação dos valores obtidos, com base nas classes definidas por Aller et al (1987).

A derradeira tarefa consistirá no cálculo do mapa DRASTIC final, o qual é obtido através da multiplicação dos índices parcelares pelas suas ponderações e adição desses resultados.

O método DRASTIC de avaliação, quantificação e posterior cartografia da vulnerabilidade dos recursos hídricos subterrâneos à contaminação foi proposto por ALLER et al em 1987, e é constituído por sete parâmetros, identificados por cada uma das letras do acrónimo DRASTIC (Figura 4). Este modelo optimiza o uso de dados já existentes para classificar áreas respectivamente aos seus potenciais de poluição, de forma a ajudar investigações directas, a prevenir custos e a estabelecer estratégias para a monitorização e remediação de sistemas (Aller et al., 1987). Ainda segundo os mesmos autores, o método DRASTIC assume que o contaminante em questão é introduzido no aquífero a partir da superfície utilizando como via de propagação a água da chuva que através da infiltração atinge o nível contaminando as águas subterrâneas, e que a área em análise terá no mínimo 100 acres $\left(\approx 405000 \mathrm{~m}^{2}\right)$.

O índice DRASTIC é calculado através da seguinte fórmula supra citada, Equação 1.

A letra de cada parâmetro representa o índice parcelar atribuído ao elemento hidrogeológico em causa e $w$ o seu peso, o qual reflecte a sua importância relativa, variando de 1 a 5 de acordo com o Quadro I.

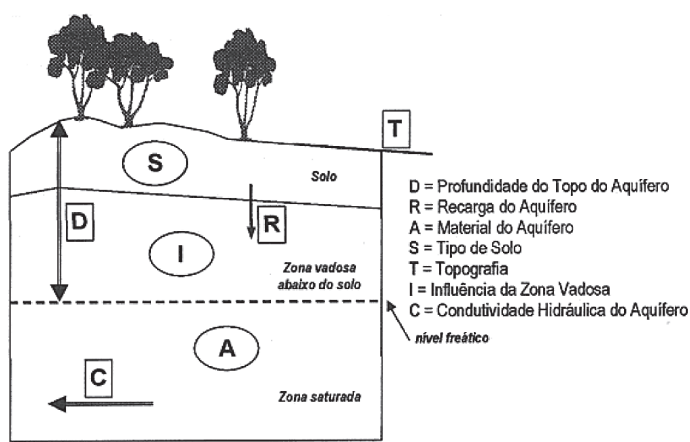

Figura 4

Parâmetros incorporados no método DRASTIC. Fonte: LNEC (2002), Lisboa

Quadro I

Peso dos parâmetros DRASTIC (Aller et al, 1987)

\begin{tabular}{llllllll}
\hline Parâmetro & D & R & A & S & T & I & C \\
\hline Peso & 5 & 4 & 3 & 2 & 1 & 5 & 3 \\
\hline
\end{tabular}




\subsubsection{Descrição dos Parâmetros DRASTIC}

$O$ índice DRASTIC «tinha como objectivo inicial ser uma ferramenta de reconhecimento mas, tem dado provas sucessivas do seu valor enquanto indicador de áreas que merecem uma avaliação hidrogeológica detalhada» (NNRC). «Apesar de ser o método para avaliar a sensibilidade aquífera mais comummente utilizado, contudo não tem intenção de prever a ocorrência/concentração de contaminação nas águas subterrâneas» (USGS, 1999).

O índice DRASTIC subdivide-se, então, em:

\section{D - Profundidade do topo do aquífero (Depth to the water table) \\ Representa a distância existente entre a super-} fície da Terra e o nível freático, nos casos em que o estudo incide sobre aquíferos livres. Quando se trata de aquíferos confinados, a profundidade é a distância ao topo do aquífero, enquanto para aquíferos semi-confinados se deve optar entre a distância ao nível freático ou ao topo do aquífero (LNEC, 2002).

No caso da bacia do Sordo este parâmetro foi estimado a partir de registos do nível hidrostático (NHE) de 41 poços inventariados por PACHECO et al (2004).

\section{R - Recarga do aquífero (net Recharge)}

«É a quantidade de água que se vai infiltrar na superfície (por ano) e atravessar a área não saturada até ao nível freático» (McGuff e Mcmullen, 2004). A recarga ocorre segundo vários processos incluindo: infiltração directa das águas de superfície; escoamento; infiltração directa da precipitação; e fluxo de águas subterrâneas (LOBO-FERreIRA, 2000).

No presente estudo de caso, a estimativa da recarga recorreu aos registos de caudal de 31 nascentes monitorizadas na bacia do rio Sordo por PACHECO e Alencoão (2006), e ao designado Método do Hidrograma descrito em Domenico e Schwartz (1990) e que pode ser sumariado do seguinte modo: se $Q_{f}\left(\mathrm{~m}^{3} / \mathrm{s}\right)$ é o caudal de base medido no final de uma recessão e $Q_{i}\left(\mathrm{~m}^{3} / \mathrm{s}\right)$ o caudal de base medido no início da recessão seguinte, então a recarga ocorrida entre recessões é: $R=\left(Q_{i}-Q_{f}\right) \frac{t_{1}}{2.3}$ em que $R\left(\mathrm{~m}^{3}\right)$ é a recarga anual e $t_{1}(\mathrm{~s})$ o tempo correspondente a um ciclo logarítmico de caudal.

\section{A - Material do Aquífero (Aquifer material)}

«Refere-se à capacidade do aquífero para atenuar os efeitos dos poluentes» (LNEC, 2002). «A dimensão do recurso e a porosidade do material do aquífero têm um grande impacto na deslocação dos contaminantes. A dimensão do recurso subterrâneo determina o tempo disponível para que diversos processos ocorram, tais como absorção, reacção e dispersão, enquanto a porosidade dos diferentes materiais aquíferos influenciam o contacto entre as substâncias poluentes e o material do aquífero» (McGuff e Mcmullen, 2004).

No caso da bacia do rio Sordo, a definição de categorias de vulnerabilidade foi feita a partir do mapa geológico (Figura 2).

\section{S - Tipo de Solo (Soil type)}

Este parâmetro tem as mesmas implicações que o material do aquífero, mas ao contrário dele que incide sobre a área saturada, este reflecte o comportamento dos contaminantes numa camada superficial de solo e saprólito com espessura normalmente inferior a $2 \mathrm{~m}$ e são quatro as propriedades de um solo que condicionam o potencial de poluição: espessura; textura; expansibilidade/ contractibilidade; e teor de matéria orgânica. (LNEC, 2002).

\section{T - Topografia (Topography)}

«A topografia refere-se ao declive do terreno. Este parâmetro condiciona a probabilidade de um poluente escoar superficialmente ou de permanecer à superfície durante o tempo suficiente para se infiltrar. Influencia ainda o desenvolvimento de solos condicionando, desta forma, o efeito de atenuação do contaminante que os mesmos normalmente apresentam» (LNEC, 2002). «Num declive de $0-2 \%$ o potencial de poluição por infiltração encontra-se no máximo, enquanto num declive superior a $18 \%$ este potencial é diminuto. Contudo, a contaminação de águas superficiais aumenta com a maior probabilidade de erosão» (Aller et al., 1987).

O declive do terreno, indispensável à definição do índice parcelar T, é calculado facilmente desde que se disponha de um software SIG e uma representação digital do relevo, por exemplo através de curvas de nível com cota associada a partir das quais se determina o Modelo Digital do Terreno (MDT) e na sequela o mapa de declives.

\section{I - Impacto da Área Não-Saturada ou Vadosa (Impact of the vadose zone)}

A área não saturada é caracterizada por um estado de saturação intermitente resultante de um nível freático oscilante (McGuff e Mcmullen, 2004). 0 impacto sobre esta área é função do «tempo de contacto entre o horizonte superficial do terreno e o poluente» (LNEC, 2002). Quanto maior for este tempo, mais extenso é o desenvolvimento de «processos de depuração: biodegradação, neutralização, filtração mecânica, reacção química, volatilização e dispersão. A fracturação nesta área é particularmente importante pois facilita a percolação vertical do poluente até ao aquífero» (LNEC, 2002). «Este parâmetro incorpora duas características, profundidade ao nível freático e a permeabilidade do solo» (McGuff e Mcmullen, 2004). 
Devido à impossibilidade de avaliar o impacto da área não saturada, recorreu-se à utilização de um índice típico, adoptando-se o valor 4 referente a "rocha ígnea e metamórfica alterada".

C - Condutividade hidráulica do aquífero (Conductivity)

$\mathrm{O}$ parâmetro $\mathrm{C}$ mede a facilidade da água subterrânea em movimentar-se através de meios permeáveis sob influência de um determinado gradiente hidráulico, a qual é controlada pela quantidade de vazios interconectados do aquífero, que podem ser poros, fracturas, cavidades ou planos de estratificação.

A condutividade hidráulica das rochas da bacia do rio Sordo foi estimada para um pequeno sector da vizinhança da Campeã com cerca de 136,4 hectares pelo método das diferenças finitas (PACHECO et al, 2004), com valores que rondaram em média $0,29 \mathrm{~m} / \mathrm{dia}$, valor bastante inferior ao limite máximo do escalão 1 para este parâmetro do DRASTIC (4,1 m/dia).

\subsection{O método Fuzzy}

ZHou et al. (1999) desenvolveram um modelo de avaliação da vulnerabilidade dos sistemas aquíferos à poluição, assente na lógica DRASTIC, que designaram padrão de reconhecimento multi-objectivos.

0 referido método tem por base a noção de que pode definir-se um local ideal como sendo o mais susceptível de ser contaminado e um outro como sendo o menos susceptível de ser contaminado, e que todos os locais se podem referir relativamente a estes dois extremos.

Se o número de locais relativamente aos quais se pretende avaliar a vulnerabilidade for $n$ e o número de parâmetros que reflectem essa mesma vulnerabilidade for $m$, então $x_{i j}$ representa o valor do parâmetro $j$ no local $i$. No seu conjunto, os valores $x_{i j}$ constituem a matriz de dados $\boldsymbol{X}$. Porém, os valores que $\mathbf{X}$ assume diferem consoante a natureza do parâmetro: quando este é definido quantitativamente ( $D, R, T, C), X$ assume os valores da variável de base (profundidade do NHE, recarga, declive, condutividade hidráulica); caso contrário $(\mathrm{A}, \mathrm{S}, \mathrm{I}) \mathrm{X}$ assume os valores dos índices atribuídos a cada parâmetro. Além disso, os parâmetros do DRASTIC são agrupados do seguinte modo: no grupo $\mathrm{A}$ a vulnerabilidade aumenta com o incremento nos valores de $\mathrm{X}$ enquanto que no grupo $\mathrm{B}$ ocorre exactamente 0 contrário. Ao grupo $A$ pertencem os parâmetros $R, A, S$, C e I, ao grupo B os parâmetros D e T.

Os valores $x_{i j}$ são de seguida normalizados relativamente aos máximos e mínimos definidos para cada uma das escalas do DRASTIC, obtendo-se os valores $r_{i j}$ : $r_{i j}= \begin{cases}\frac{x_{i j}-x_{\operatorname{minj}}}{x_{\operatorname{maxj}}-x_{\min j}} & x_{i j} \in \text { grupo A } \\ 1-\frac{x_{i j}-x_{\min j}}{x_{\operatorname{maxj}}-x_{\min j}} & x_{i j} \in \text { grupo B }\end{cases}$

Se $r_{i j}=1$ então o local i é o mais susceptível de ser contaminado no parâmetro $\mathrm{j}$ enquanto que se $r_{i j}=$ 0 o local i é o menos susceptível de ser contaminado nesse mesmo parâmetro. Admita-se que existe um local relativamente ao qual $r_{i j}=1$ e um outro relativamente ao qual $r_{i j}=0$, para qualquer $\mathrm{j}$, e designem-se esses locais por $\mathbf{g}$ e $\mathbf{b}$, respectivamente. Nestas circunstâncias, o estudo da proximidade dos locais em estudo relativamente a estes dois extremos converte-se num problema de lógica fuzzy.

As distâncias Euclidianas entre um local $\mathbf{i}$ e os locais $\mathrm{g}$ e b são dadas por:

$d_{i g}=\sqrt{\sum_{j=1}^{m}\left[w_{j}\left(g_{i}-r_{i j}\right)\right]^{2}}$

$d_{i b}=\sqrt{\sum_{j=1}^{m}\left[w_{j}\left(r_{i j}-b_{i}\right)\right]^{2}}$

em que $d_{i g}$ e $d_{i b}$ são as referidas distâncias e $w_{j}$ é o peso atribuído ao parâmetro $\mathrm{j}$ de acordo com as regras do DRASTIC. A projecção gráfica da relação entre o local i e os locais $\mathbf{g}$ e b apresenta-se na Figura 5.

Se a posição óptima do local i relativamente ao local ideal $\mathbf{g}$ for designada por $u_{i}$ então a posição óptima relativamente ao local b é $1-u_{i}$. Na lógica fuzzy, $u_{i} e$ $1-u_{i}$ podem ser encarados como pesos, de modo que $D_{i}$ $=u_{i} d_{i g}$ e $D_{i b}=\left(1-u_{i}\right) d_{i b}$ são as distâncias ponderadas de $\mathbf{i}$ a $\mathbf{g}$ e a b. A determinação de $u_{i}$ passa pela minimização da função objectiva + que graficamente corresponde à projecção de $\mathbf{i}$ sobre a recta que une os pontos $\mathbf{g}$ e $b$ (Figura 6) e analiticamente à resolução da equação:

$\frac{d\left(D_{i g}^{2}+D_{i b}^{2}\right)}{d u_{i}}=0$

do que resulta:

$u_{i}=\left[1+\frac{\sum_{j=1}^{m}\left(w_{j}\left(r_{i j}-1\right)\right)^{2}}{\sum_{j=1}^{m}\left(w_{j} r_{i j}\right)^{2}}\right]^{-1}$

Os valores $u_{i}$ representam o grau de vulnerabilidade do meio relativamente à contaminação das águas subterrâneas, de acordo com o modelo fuzzy do padrão de reconhecimento multi-objectivos. Esse grau varia desde os extremos g (o local mais fácil de ser contaminado) e b (local mais difícil de ser contaminado), de acordo com os escalões apresentados no Quadro II.

A avaliação da vulnerabilidade à contaminação na bacia do rio Sordo, pelo método DRASTIC na sua versão original, utilizou dados muito diversificados, de acordo com a sua origem.

No sentido de se uniformizar a tipologia de dados para a avaliação da vulnerabilidade de acordo com 


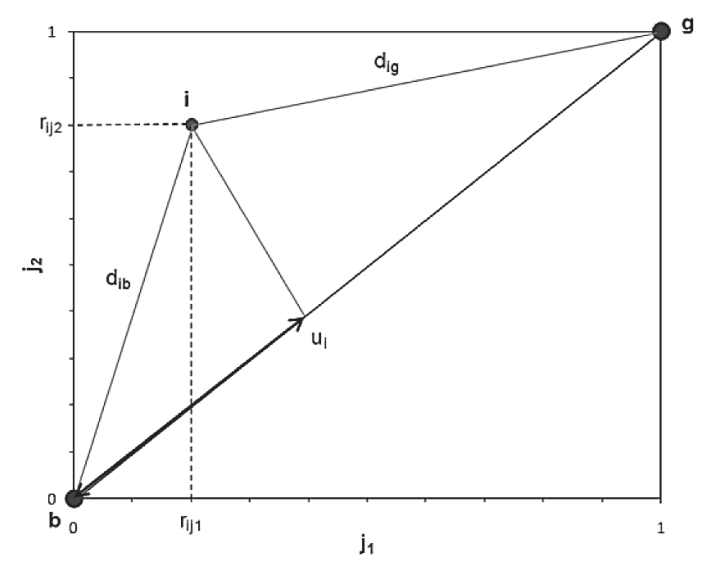

Figura 5

Ilustração da determinação da posição óptima do local i relativamente aos locais $\mathrm{g}$ e b, designada por $u_{i}$, considerando somente duas variáveis do DRASTIC.

\section{Quadro II}

Avaliação da vulnerabilidade de acordo com o modelo fuzzy do padrão de reconhecimento multi-objectivos (ZHou et al, 1999)

\begin{tabular}{|c|c|}
\hline Grau de Vulnerabilidade & Decisão \\
\hline$<0,2$ & Muito difícil de ser contaminado \\
\hline $0,2-0,4$ & Difícil de ser contaminado \\
\hline $0,4-0,6$ & Pode ser contaminado \\
\hline $0,6-0,8$ & Fácil de ser contaminado \\
\hline $0,8-1$ & Muito fácil de ser contaminado \\
\hline
\end{tabular}

o modelo fuzzy do padrão de reconhecimento multiobjectivos, optou-se por sobrepor uma grelha de pontos (208) regularmente espaçados $(500 \mathrm{~m} \times 500 \mathrm{~m})$ ao mapa de cada variável e determinar o valor da mesma em cada ponto utilizando uma ferramenta de amostragem do ArcGIS (ArcToolBox > Spatial Analyst Tools > Extraction > Sample). Os resultados correspondem no seu conjunto à matriz $\mathrm{X}$. Usando esta matriz como matriz de dados, calcularam-se os valores de $u_{i}$. De modo a compararem-se os resultados do modelo fuzzy com os resultados do modelo original, normalizaram-se os últimos tomando em consideração os valores mínimo (26) e máximo (226) do DRASTIC:

DRASTIC NORMALIZADO = (DRASTIC - 26) / (226 - 26) (6)

Através dos resultados da comparação verificase que as vulnerabilidades calculadas pelo modelo $f u$ zzy são sistematicamente superiores às estimadas pelo DRASTIC original, em média $6,3 \%$.

\subsection{Método das Concentrações em Nitratos}

O modelo DRASTIC estabelece a vulnerabilidade intrínseca do meio à contaminação, não existindo muitos trabalhos que aproximem o método a uma análise de risco, sendo a principal fragilidade a sua subjectividade inerente à conversão das escalas físicas na escala de 10 categorias e na atribuição dos pesos relativos a cada parâmetro.

Com base em todos os pressupostos expostos anteriormente para este método, segue-se a sua explicação. A adição do parâmetro L (Uso do Solo), permite identificar as fontes potenciais de contaminação antrópica na bacia hidrográfica do rio Sordo, relacionam-se fundamentalmente com actividades agro-pecuárias, domésticas e industriais, cuja distribuição espacial se pôde identificar na Figura 3.

Constatou-se que cerca de $32 \%$ da região apresenta uma vulnerabilidade específica muito reduzida (escalão 1), 59\% reduzida (escalões 3 e 4), 3\% moderada (escalão 5), e 6\% muito elevada (escalões 9 e 10). Ou seja, verifica-se que a vulnerabilidade específica é reduzida em mais de $90 \%$ da área, pelo que o risco real de poluição antevê-se baixo.

Para o cálculo do risco potencial, conhecidos os valores do DRASTIC na versão original, aplica-se a seguinte equação:

RISCO POTENCIAL = DRASTIC $+4 L$

Desse modo, os valores máximo e mínimo do risco potencial são 27 e 266 , respectivamente, pelo que a obtenção de um mapa normalizado deste parâmetro passa pela aplicação da fórmula:

RISCO POTENCIAL NORMALIZADO $=\frac{\text { RISCO POTENCIAL-27 }}{239}$

\subsubsection{Revisão das Escalas dos Parâmetros e dos} Respectivos Pesos

A revisão das escalas de cada parâmetro passa por pesar o valor atribuído a cada local com base na concentração em nitratos registada ou estimada para esse local, da seguinte forma:

$P^{*}=\frac{P_{\max } \times\left(\mathrm{NO}_{\max }-\mathrm{NO}_{\min }\right)-\left(P_{\max }-P_{\min }\right) \times\left(\mathrm{NO}_{\max }-\mathrm{NO}\right)}{N O 3_{\max }-\mathrm{NO}_{\min }}$

- $P^{*}$ é o valor modificado do parâmetro $P(D, R$, A, S, T, I, C ou L), sendo $P_{\max }$ e $P_{\text {min }}$ os valores máximo e mínimo atribuídos à região pelo DRASTIC;

- NO3 é a concentração em nitratos registada ou estimada para um dado local, sendo $\mathrm{NO}_{\text {max }}$ e $\mathrm{NO}_{\text {min }}$ os valores máximo e mínimo dessas concentrações, registados na região num dado momento.

A revisão dos pesos é conseguida através da análise de correlação entre os valores de cada parâmetro nos diversos locais em análise e as respectivas concentrações em nitrados, seguida da redefinição dos pesos com base na hierarquia dessas correlações, numa lógica idêntica à revisão das escalas de cada parâmetro. 
A fórmula utilizada na revisão dos pesos atribuídos a cada parâmetro é a seguinte:

$$
W^{*}=\frac{W_{\max } \times\left(C_{\max }-C_{\min }\right)-\left(W_{\max }-W_{\min }\right) \times\left(C_{\max }-C\right)}{C_{\max }-C_{\min }}
$$

- W* é o valor modificado do peso $\mathrm{W}$ atribuído ao parâmetro $P(D, R, A, S, T, I, C$ ou $L)$, sendo $\mathrm{W}_{\max }$ e $\mathrm{W}_{\min }$ os valores máximo (5) e mínimo (1) atribuídos pelo DRASTIC.

- C é a correlação entre o parâmetro P e a concentração em nitratos, estimada para a totalidade da região, sendo $C_{\max }$ e $C_{\min }$ os valores máximo e mínimo dessas correlações.

À semelhança do que se observou com a redefinição dos valores de cada parâmetro em cada ponto da região em estudo (eq. 9), a aplicação da Equação 10 também possibilita uma reapreciação da importância de cada parâmetro na avaliação do risco de poluição, função da sua correlação com a concentração em nitratos.

O cálculo das correlações deve contemplar o facto dos parâmetros do modelo DRASTIC na versão original serem variáveis categóricas enquanto que as concentrações em nitratos constituem variáveis medidas numa escala contínua. Devem por isso utilizar-se métodos não paramétricos no cálculo dos coeficientes de correlação, como por exemplo o algoritmo de Spearman ou o algoritmo Kendall Tau.

Constata-se que, com excepção dos escalões inferiores de cada parâmetro, a redefinição dos parâmetros teve por consequência uma clara diminuição dos valores $\mathrm{P}^{*}$ relativamente aos correspondentes valores de $P$, pelo que se conclui que o risco real de contaminação das águas subterrâneas é claramente inferior ao risco potencial. A tal não será alheio o facto das concentrações em nitratos serem generalizadamente bastante reduzidas (Figura 10).

Os resultados relativos à redefinição dos pesos atribuídos a cada parâmetro do DRASTIC apresentam-se no Quadro III. Os pesos originais surgem na $2^{\mathrm{a}}$ coluna, os coeficientes de correlação de Spearman e Kendall Tau nas $3^{\mathrm{a}}$ e $4^{\mathrm{a}}$ e os respectivos pesos ajustados (Equação 10) nas $5^{a}$ e $6^{a}$. Finalmente, a média dos dois pesos ajustados surge na $7^{\mathrm{a}}$ e última coluna. Não foi possível redefinir os pesos do I e do C pelo facto destes parâmetros apresentarem valores constantes em toda a bacia, e por essa via não apresentarem qualquer correlação com a concentração em nitratos. Na impossibilidade de reapreciar a importância dos parâmetros I e C no âmbito da análise do risco real de contaminação das águas subterrâneas da bacia hidrográfica do rio Sordo, optouse por manter os pesos originais.

\section{Resultados}

\subsection{Cálculo do Índice DRASTIC}

O cálculo do índice DRASTIC relativo à bacia hidrográfica do rio Sordo recorreu ao software SIG denominado ArcGIS da ERSI e a informação digital diversa abrangendo os diversos parâmetros do modelo.

Aplicando a equação 1 aos índices parcelares, recorrendo para tal à ferramenta Raster Calculator do módulo Spatial Analyst, obtém-se o mapa de vulnerabilidade DRASTIC relativo à bacia hidrográfica do Sordo, que se apresenta a Figura 6, com valores de vulnerabilidade intrínseca reduzida a moderada (escalões de 80-99, 100-119 e 120-139).

\subsection{Cálculo do modelo fuzzy}

As distribuições espaciais das vulnerabilidades DRASTIC relativas ao modelo original normalizado e ao modelo fuzzy apresentam-se nas Figuras 7 e 8 . No primeiro caso, as vulnerabilidades enquadram-se com os escalões "difícil de ser contaminado" e "pode ser contaminado", basicamente reproduzindo os escalões "80-99" e "100-119" + "120-139" do modelo DRASTIC original não normalizado (Figura 6).

Quadro III

Redefinição dos pesos dos parâmetros D, R, A, S, T, e L, pelo Método da Concentração em Nitratos, na área da bacia hidrográfica do rio Sordo

\begin{tabular}{|c|c|c|c|c|c|c|}
\hline Parâmetro & Peso Original & $\begin{array}{l}\text { Coeficiente de } \\
\text { Spearman }\end{array}$ & $\begin{array}{l}\text { Coeficiente de } \\
\text { Kendal Tau }\end{array}$ & $\begin{array}{c}\text { Peso Ajustado pelo Coeficiente } \\
\text { de Spearman }\end{array}$ & $\begin{array}{l}\text { Peso Ajustado pelo } \\
\text { Coeficiente de Kendal Tau }\end{array}$ & $\begin{array}{l}\text { Peso Ajustado } \\
\text { Médio }\end{array}$ \\
\hline D & 5 & 0,390 & 0,310 & 5,0 & 5,0 & 5,0 \\
\hline $\mathrm{R}$ & 4 & $-0,328$ & $-0,249$ & 1,0 & 1,0 & 1,0 \\
\hline$A$ & 3 & 0,000 & 0,001 & 2,8 & 2,8 & 2,8 \\
\hline $\mathrm{S}$ & 2 & 0,114 & 0,085 & 3,5 & 3,4 & 3,4 \\
\hline $\mathrm{T}$ & 1 & 0,142 & 0,134 & 3,8 & 3,7 & 3,8 \\
\hline 1 & 5 & nd & nd & 5,0 & 5,0 & 5,0 \\
\hline $\mathrm{C}$ & 3 & nd & nd & 3,0 & 3,0 & 3,0 \\
\hline $\mathrm{L}$ & 4 & 0,143 & 0,104 & 3,6 & 3,5 & 3,6 \\
\hline Mínimo & 1 & $-0,33$ & $-0,25$ & 1,0 & 1,0 & 1,0 \\
\hline Máximo & 5 & 0,39 & 0,31 & 5,0 & 5,0 & 5,0 \\
\hline
\end{tabular}




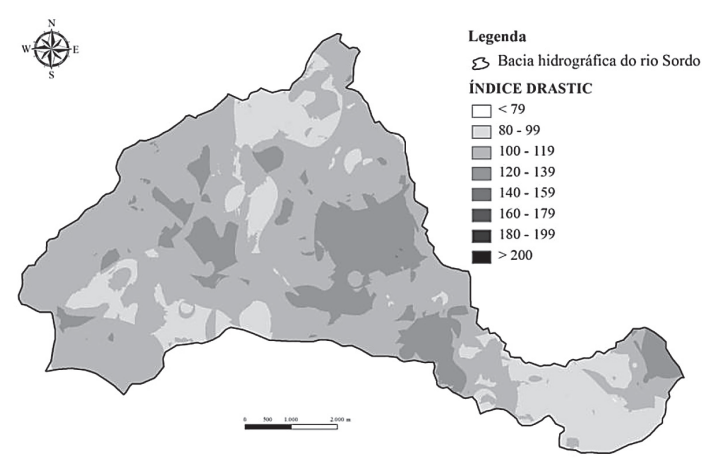

Figura 6

Vulnerabilidade da bacia do Sordo com base no Índice DRASTIC

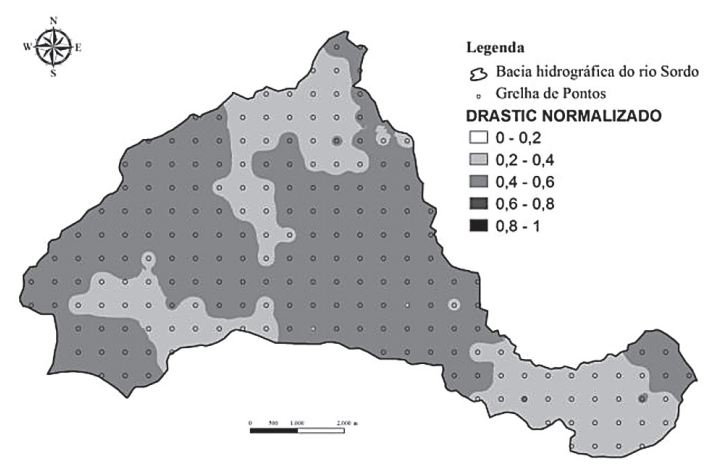

Figura 7

Mapa de vulnerabilidade DRASTIC (modelo original normalizado)

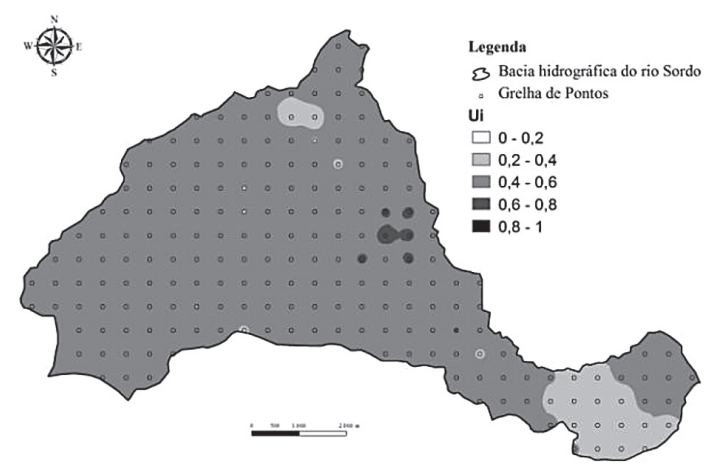

Figura 8

Mapa de vulnerabilidade DRASTIC (modelo fuzzy)

0 incremento sistemático dos valores $u_{i}$, relativamente aos valores homólogos do DRASTIC original normalizado, tem expressão espacial significativa. Neste contexto, grande parte da área passa a ser classificada como área que pode ser contaminada, persistindo duas pequenas manchas classificadas como áreas difíceis de ser contaminadas, mas surgindo na área de contacto entre os xistos e os granitos pequenas manchas de áreas classificadas como fáceis de ser contaminadas.

\subsection{Cálculo do método da concentração em nitratos}

O mapa do risco potencial normalizado referente à bacia do rio Sordo ilustra-se na Figura 36. Relativamente ao mapa do DRASTIC normalizado (Figura 7), constata-se que as áreas de vulnerabilidade intrínseca moderada localizadas no sector Oeste da bacia, correspondentes à área de cabeceira do curso de água principal, não constituem áreas de risco potencial moderado ou elevado dada a ocupação florestal que se regista nessas áreas. A ocupação florestal manifesta-se dessa forma como um factor de redução do risco de contaminação das águas subterrâneas nos locais onde a vulnerabilidade intrínseca suscita uma preocupação razoável.

A distribuição das concentrações em nitratos, no período de recolha das amostras, apresenta-se na Figura 10. Verifica-se que as concentrações são reduzidas (< $5 \mathrm{mg} / \mathrm{l}$ ) em grande parte da bacia, com excepção do sector Este e do bordo Sul do sector central onde por vezes se atingem valores superiores a $20 \mathrm{mg} / \mathrm{l}$ e num local se registou um valor superior a $75 \mathrm{mg} / \mathrm{l}$.

Os resultados sugerem que o efeito de diluição do nitrato que as recargas elevadas produzem supera o efeito do transporte promovido por esses mesmos volumes de água, obtendo-se como resultado global uma redução do risco real traduzido por concentrações em nitratos reduzidas em grande parte da área da bacia. Põem também em evidência o efeito que a presença de relevos aplanados pode ter na promoção da infiltração e consequentemente no surgimento de focos de contaminação de águas subterrâneas.

A representação espacial do risco real de contaminação das águas subterrâneas na bacia hidrográfica do rio Sordo apresenta-se na Figura 11, tendo os valores do risco sido normalizados utilizando fórmulas idênticas às Equações 7 e 10 mas considerando que o risco real

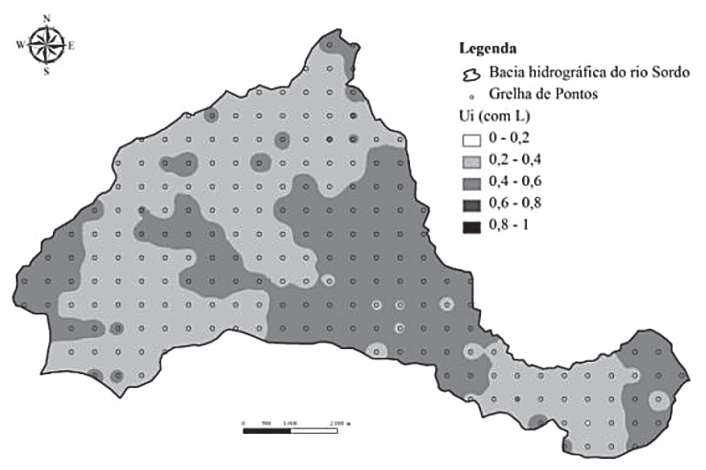

Figura 9

Mapa normalizado da vulnerabilidade DRASTIC, com inclusão do Parâmetro L 


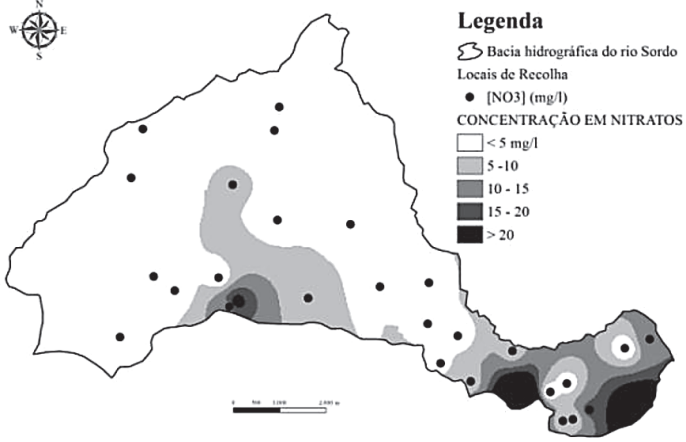

Figura 10

Concentração em nitratos em Outubro de 2002 na bacia hidrográfica do rio Sordo

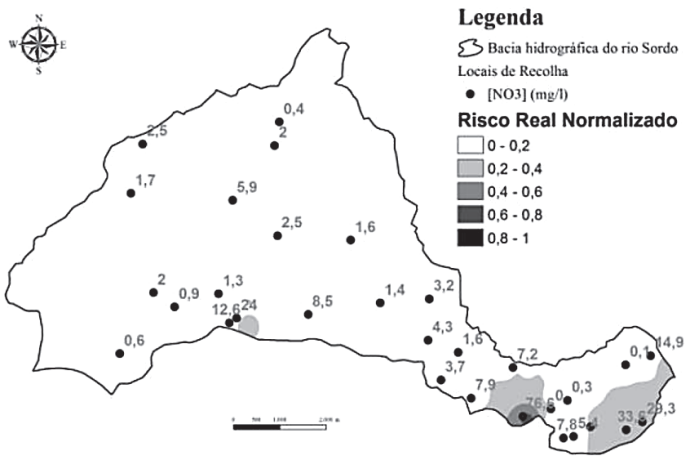

Figura 11

Mapa normalizado da vulnerabilidade DRASTIC, com inclusão do Parâmetro L (Método da Concentração de Nitratos)

varia em termos absolutos entre 30.4 e 274.7 em consonância com os pesos ajustados médios representados no Quadro III.

A realidade do momento em que foram colhidas as amostras de água e analisadas as respectivas concentrações em nitratos (Outubro de 2002) revela uma situação de risco real diminuto na maior parte da área da bacia, podendo identificar-se três pequenas manchas onde esse risco é reduzido. Não obstante, uma nascente na vizinhança de Arnadelo, sobranceira à albufeira da barragem do rio Sordo, constituía um local de risco real elevado. Tomando em consideração a concentração em $\mathrm{NO}_{3}$ (76.6 mg/l) dessa nascente, configurou-se nesse local, ao tempo, um cenário de contaminação efectiva.

\section{5 - Conclusões}

Os resultados do modelo DRASTIC, na versão original, atribuem uma vulnerabilidade intrínseca re- duzida a moderada à bacia hidrográfica do rio Sordo, integrando a totalidade da mesma nos escalões 80-99, 100-119 e 120-139.

Comparando os mapas das Figuras 6, 7 e 8, pode concluir-se que: 1) as vulnerabilidades normalizadas enquadram-se com os escalões "difícil de ser contaminado" e "pode ser contaminado" (Quadro I), basicamente reproduzindo os escalões "80-99" e "100-119" + "120-139" do modelo DRASTIC original; 2) as vulnerabilidades calculadas pelo modelo fuzzy-DRASTIC são sistematicamente superiores às estimadas pelo DRASTIC normalizado, em média 6,3\%. Nesse contexto, grande parte da área passa a ser classificada como área que pode ser contaminada, persistindo duas pequenas manchas classificadas como áreas difíceis de ser contaminadas, mas surgindo na área de contacto entre os xistos e os granitos pequenas manchas de áreas classificadas como fáceis de ser contaminadas.

A vulnerabilidade específica, estimada pelo método da concentração em nitratos através da adição do parâmetro L (uso do solo) ao modelo DRASTIC original, distribui-se do seguinte modo pela bacia hidrográfica do rio Sordo: cerca de $32 \%$ da região apresenta uma vulnerabilidade específica muito reduzida, 59\% reduzida, 3\% moderada, e $6 \%$ muito elevada.

O risco potencial, definido como a soma da vulnerabilidade intrínseca (resultados do DRASTIC original) pela vulnerabilidade específica (parâmetro L), sugere que as áreas de vulnerabilidade intrínseca moderada localizadas no sector Oeste da bacia, correspondentes à área de cabeceira do curso de água principal, não constituem áreas de risco potencial moderado ou elevado dada a ocupação florestal que se regista nessas áreas. A ocupação florestal manifesta-se dessa forma como um factor de redução do risco de contaminação das águas subterrâneas nos locais onde a vulnerabilidade intrínseca suscita uma preocupação razoável.

A realidade da bacia, aferida em Outubro de 2002, revela uma situação de risco real diminuto na maior parte da sua área de abrangência. Não obstante, uma nascente na vizinhança de Arnadelo, sobranceira à albufeira da barragem do rio Sordo, constituía nessa altura um local de risco real elevado.

\section{6 - Referências Bibliográficas}

Afshar, A.; Mariño, M. A.; Asce, H. M.; Ebtehaj, M. e Moosavi, J. (2007) - "Rule-based fuzzy system for assessing groundwater vulnerability". Journal of Environmental Engineering, Maio, pp. 532-540. 
Agroconsultores e Coba (1991) - Carta de solos, carta do uso actual da terra e carta de aptidão da terra do nordeste de Portugal. Relatório Técnico, Universidade de Trás-osMontes e Alto Douro, Vila Real, 311 p.

Antonakos, A. K. e Lambrakis, N. J. (2007) - "Development and testing of three hybrid methods for assessment of aquifer vulnerability to nitrates, based on the DRASTIC model, an example from NE Korinthia, Greece". Journal of Hydrology $n^{\circ} 333$, Estados Unidos da América, pp. 288-304.

Aller, L.; Bennett, T.; Lehr, J. H.; Petty, R. e Hackett, G. (1987) - DRASTIC: a standardized system for evaluating ground water pollution potential using hydrogeologic settings. Environmental Protection Agency (EPA Number: 600287035), USA, $643 \mathrm{p}$.

Domenico, P. e Schwartz, F. (1990) - Physical and Chemical Hydrogeology. John Wiley \& Sons, New York, USA, 824 p.

ESRI (2006) - ArcMap (version 9.2) manual. New York St., Redlands, USA, 131p.

Kalinski, R.; Kelly, W.; Bogard, I.; Ehrman, R.e Yamamoto, P. (1994) - Correlation between DRASCTIC vulnerabilities and incidents of VOC contamination of municipal wells in Nebraska. In: Ground Water 32 (1), pp. 31-34.

Laboratorio Nacional de Engenharia Civil (LNEC) (2002) - Cartografia da Vulnerabilidade à poluição das águas subterrâneas do concelho de Montemor-o-Novo utilizando o método DRASTIC. LNEC, Departamento de Hidráulica, Grupo de Investigação de Águas Subterrâneas, Lisboa, Proc.607/1/14252, Março 2002.

Lobo-Ferreira, J. P. (2000) - "Aquifer recharge and evaluation of groundwater vulnerability to pollution". Coastin, n. ${ }^{\circ} 2$, New Delhi, pp. 8-9.
Mclay, C. D. A.; Dragden, R.; Sparling, G. e Selvarajah, N. (2001) - Predicting groundwater nitrate concentrations in a region of mixed agricultural land use: a comparison of three approaches. In: Environmental Pollution 115, pp. 191-204.

McGuff, H. e McMullen, A. (2004) - Exploring Aquifer Vulnerability for Hornby Island BC. In: GIS 470 term project, University of British Columbia. Canadá.

Nebraska Natural Resources Commission (NNRC) - DRastic Methodology.

Pacheco, F. A. L. e Alencoão, A. M. P. (2006) - "Role of fractures in weathering of solid rocks: narrowing the gap between experimental and natural weathering rates". Journal of Hydrology, n. ${ }^{\circ} 316$, Estados Unidos da América, pp 248-265.

Pacheco, F. A. L.; Sousa Oliveira, A.; Alencoão, A. M. P.; Ribeiro, I. C. e FARIA, M. J. (2004) - "Avaliação da vulnerabilidade à contaminação de águas subterrâneas pelo método DRASTIC na bacia hidrográfica do rio Sordo". $7^{\circ}$ Congresso da Água: Água - Qualidade de Toda a Vida, 8-12 de Março, Lisboa. Resumos de Comunicações, p. 31 (comunicação alargada em CD, 12p.).

United States Geological Survey (UGSG) (1999) - Improvements to the DRASTIC ground-water vulnerability mapping method. USGS Fact Sheet FS-066-99.

ZADEH, L. A. (1965) - "Fuzzy sets". Information and Control, vol. 8, n. ${ }^{\circ} 3, \mathrm{pp} 338-353$.

Zhou, H.; Wang, G. e Yang, Q. (1999) - "A multi-objective fuzzy pattern recognition model for assessing groundwater vulnerability based on the DRASTIC system". Hydrological Sciences Journal, vol. 44, $n^{\circ}$ 4, pp. 611-618. 